Supplementary Information

\title{
Synthesis of $\mathrm{Au} @ \mathrm{Cu}_{2} \mathrm{O}$ Core-Shell Nanoparticles with Tunable Shell Thickness and Their Degradation \\ Mechanism in Aqueous Solutions
}

Shilei Zhu, ${ }^{1}$ Dan Deng, ${ }^{1}$ Mai Thanh Nguyen, ${ }^{1}$ Yuen-ting Rachel Chau, ${ }^{1}$ Cheng-Yen Wen, ${ }^{2}$ and Tetsu Yonezawa ${ }^{1,3, *}$

${ }^{1}$ Division of Materials Science and Engineering, Faculty of Engineering, Hokkaido University, Kita 13 Nishi 8, Kita-ku, Sapporo, Hokkaido 060-8628, Japan

${ }^{2}$ Department of Materials Science and Engineering, National Taiwan University, No. 1, Section 4, Roosevelt Rd., Da'an District, Taipei, 10617, Taiwan

${ }^{3}$ Institute for the Promotion of Business-Regional Collaboration, Hokkaido University, Kita 21, Nishi 11, Kita-ku, Sapporo, Hokkaido 001-0021, Japan

*Email: tetsu@eng.hokudai.ac.jp

Synthesis of Au NPs: $500 \mathrm{~mL}$ of $0.5 \mathrm{mM} \mathrm{HAuCl}_{4}\left(\mathrm{HAuCl}_{4} \cdot 3 \mathrm{H}_{2} \mathrm{O}, 0.1030 \mathrm{~g}\right)$ aqueous solution was heated to boiling in a $1 \mathrm{~L}$ Erlenmeyer flask under vigorous stirring (1000 rpm). Afterward, a pre-heated $25 \mathrm{~mL}$ of a $1 \mathrm{wt} . \%$ aqueous solution of trisodium citrate dihydrate $(0.25 \mathrm{~g})$ was added rapidly with stirring $(1000 \mathrm{rpm})$ and boiling for $15 \mathrm{~min}$ before slowly cooling down to room temperature $\left(25^{\circ} \mathrm{C}\right)$.

S1 / S7 
Synthesis of Ag NPs: $450 \mathrm{~mL}$ of $1 \mathrm{mM} \mathrm{AgNO} 3$ was heated to the boiling point, and then $30 \mathrm{~mL}$ of pre-warmed $1 \mathrm{wt} \%$ trisodium citrate was quickly added and boiling for $30 \mathrm{~min}$ before slowly cooling down to room temperature $\left(25^{\circ} \mathrm{C}\right)$.
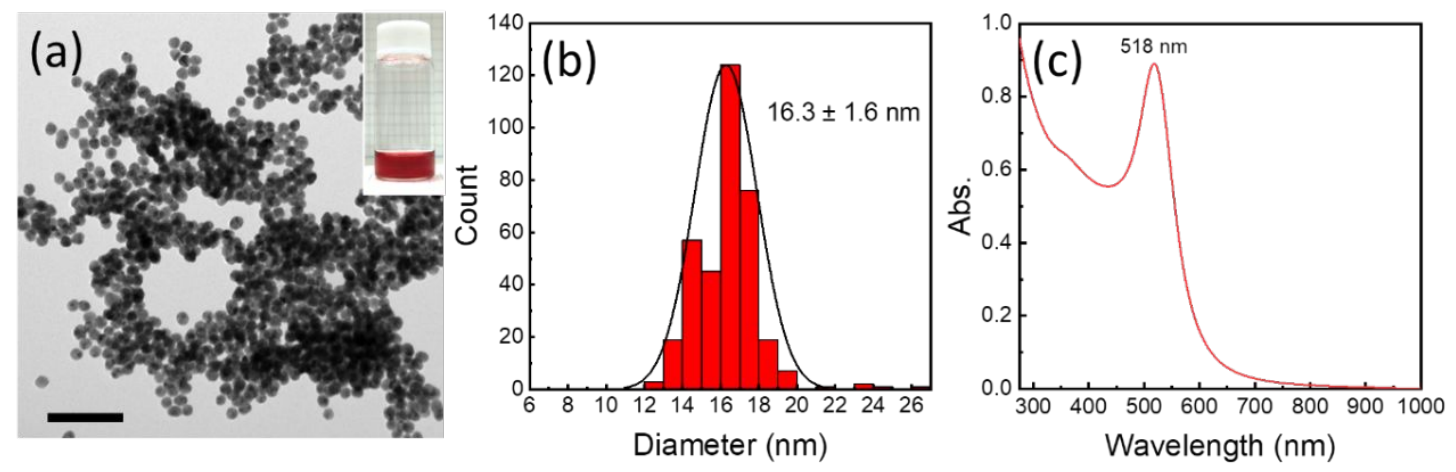

Figure S1 (a) TEM image (scale bar: $100 \mathrm{~nm}$ ) with inset photo (b) size histogram and (c) UV-Vis absorption spectra of as-synthesized Au NPs.

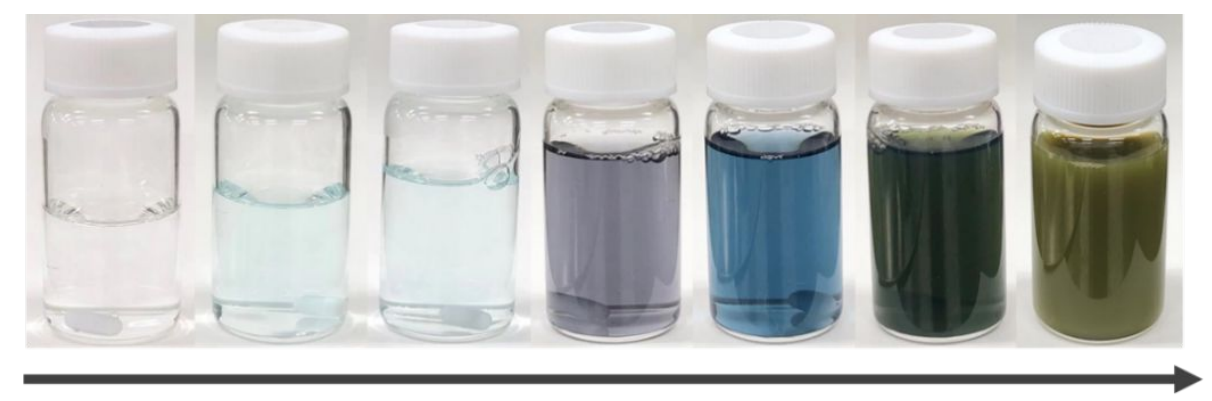

Figure $\mathbf{S 2}$ Color change in the typical synthesis of $\mathrm{Au} @ \mathrm{Cu}_{2} \mathrm{O}$ NPs. 
a

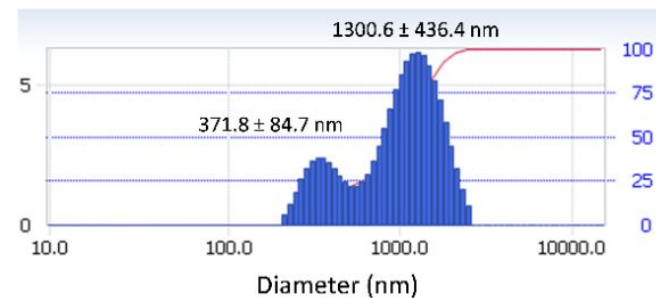

C

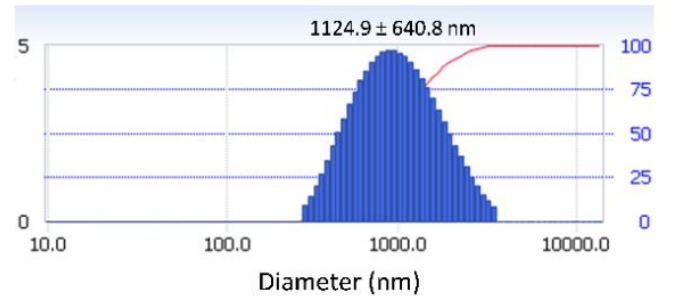

b

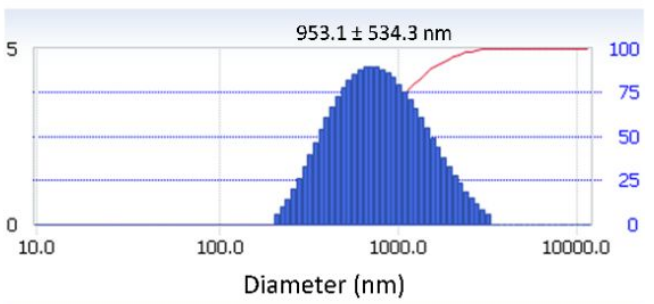

d

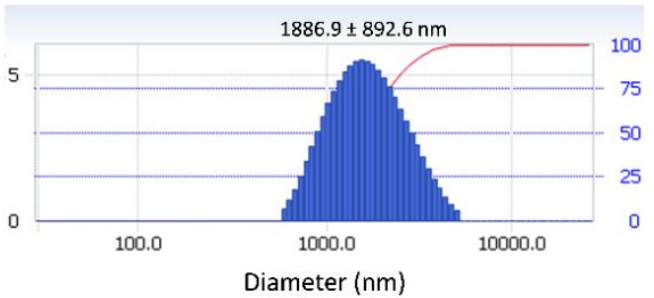

Y axis: left: Scattering intensity distribution (\%); right: Accumulated frequency distribution (\%)

Figure S3. DLS results of as-synthesized $\mathrm{Au} @ \mathrm{Cu}_{2} \mathrm{O}$ core-shell nanoparticles: a) $\left.\left.\left.\mathrm{Au} @ \mathrm{Cu}_{2} \mathrm{O}-100, b\right) \mathrm{Au} @ \mathrm{Cu}_{2} \mathrm{O}-10, \mathrm{c}\right) \mathrm{Au} @ \mathrm{Cu}_{2} \mathrm{O}-2, \mathrm{~d}\right) \mathrm{Au} @ \mathrm{Cu}_{2} \mathrm{O}-1$ (Concentration: 0.1 $\mathrm{mM}$ (based on $\mathrm{Au}$ ); Solvent: ethanol).

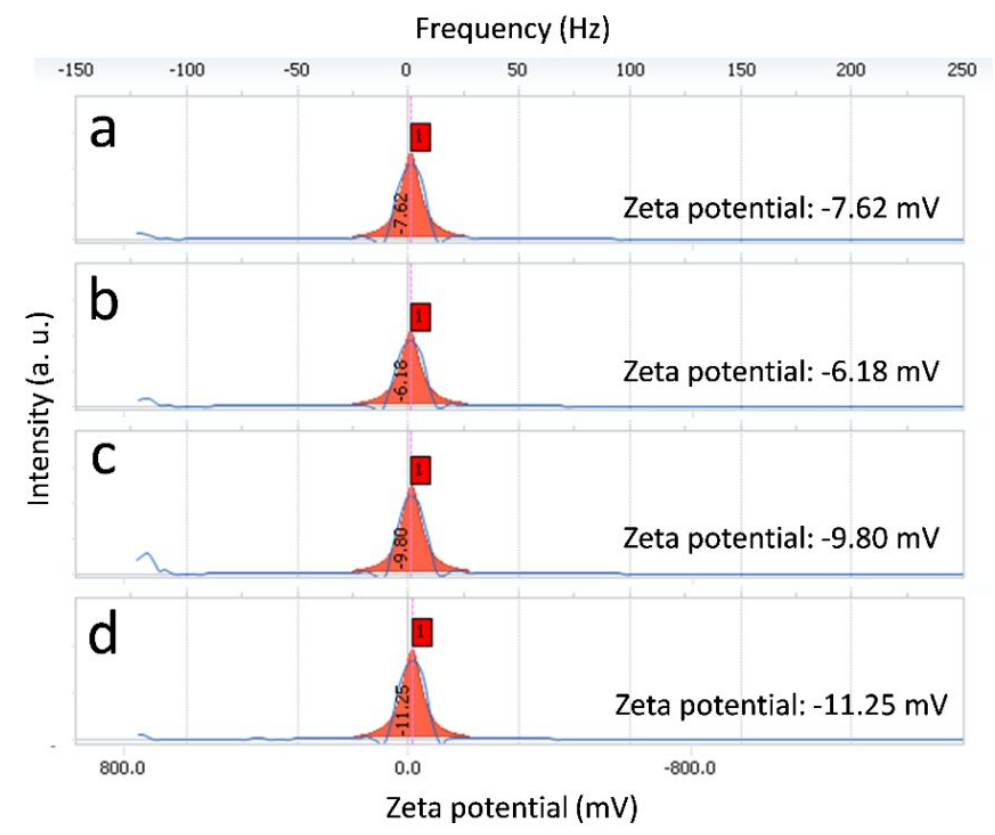

Figure S4. Zeta potential results of as-synthesized $\mathrm{Au} @ \mathrm{Cu}_{2} \mathrm{O}$ core-shell nanoparticles: a) $\left.\left.\left.\mathrm{Au} @ \mathrm{Cu}_{2} \mathrm{O}-100, \mathrm{~b}\right) \mathrm{Au} @ \mathrm{Cu}_{2} \mathrm{O}-10, \mathrm{c}\right) \mathrm{Au} @ \mathrm{Cu}_{2} \mathrm{O}-2, \mathrm{~d}\right) \mathrm{Au} @ \mathrm{Cu}_{2} \mathrm{O}-1$. (Concentration: 0.1 $\mathrm{mM}$ (based on $\mathrm{Au}$ ); Solvent: ethanol). 


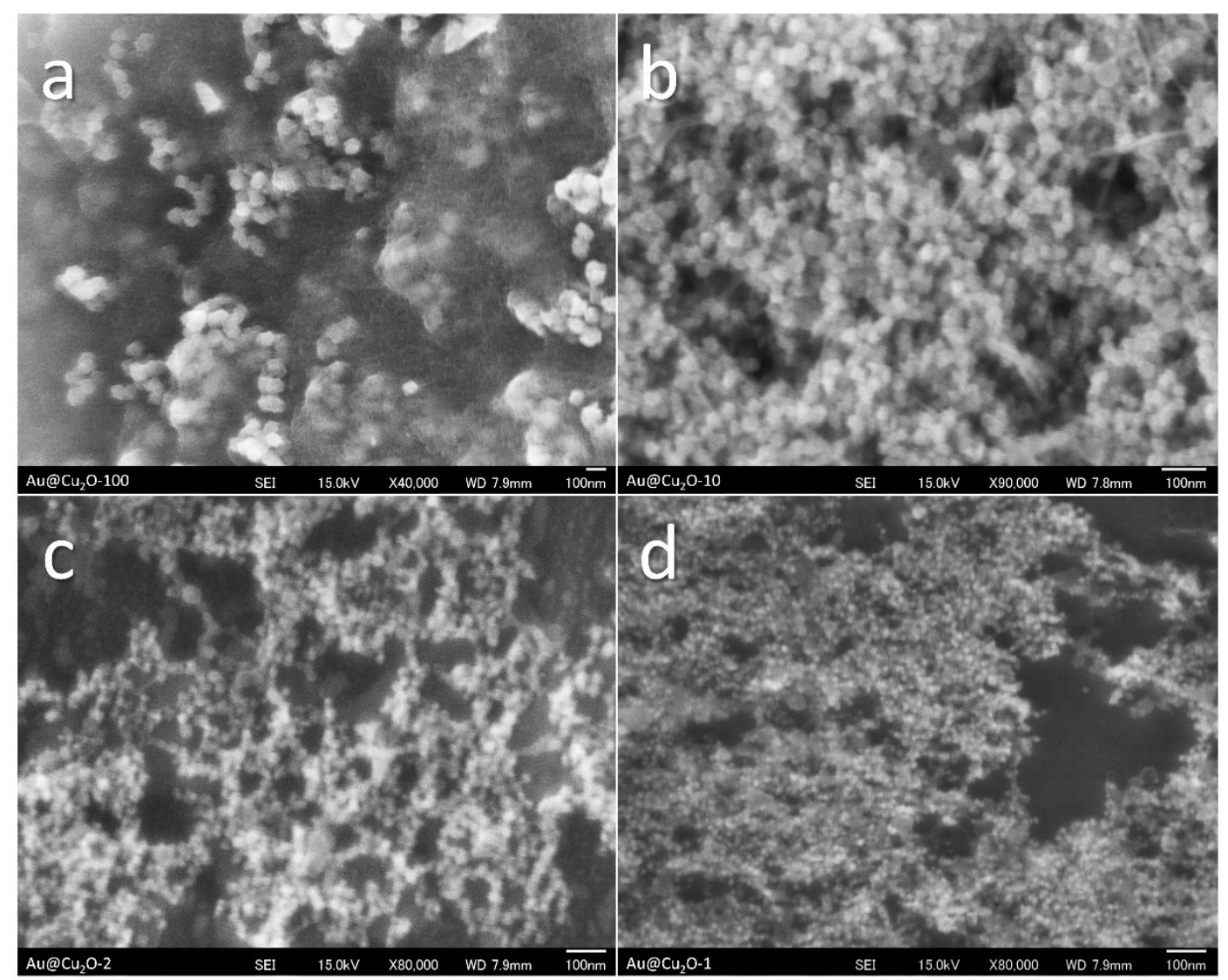

Figure S5 SEM images of as-synthesized $\mathrm{Au} @ \mathrm{Cu}_{2} \mathrm{O}$ core-shell nanoparticles: a) $\mathrm{Au} @ \mathrm{Cu}_{2} \mathrm{O}-100$, b) $\mathrm{Au} @ \mathrm{Cu}_{2} \mathrm{O}-10$, c) $\left.\mathrm{Au} @ \mathrm{Cu}_{2} \mathrm{O}-2, \mathrm{~d}\right) \mathrm{Au} @ \mathrm{Cu}_{2} \mathrm{O}-1$. 


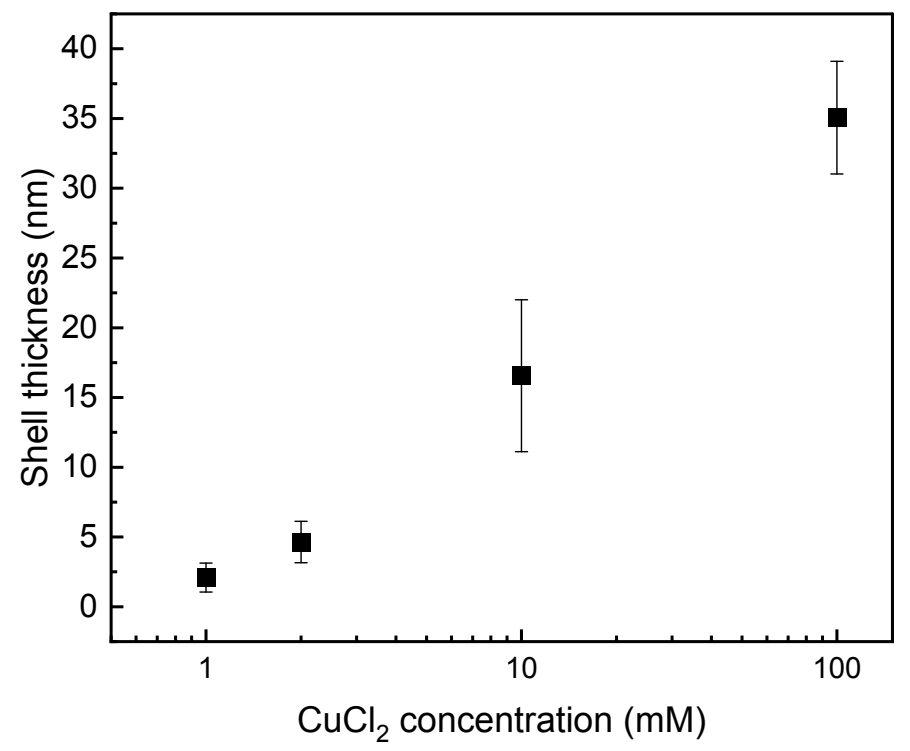

Figure S6 Increasing $\mathrm{Cu}_{2} \mathrm{O}$ shell thickness of $\mathrm{Au} @ \mathrm{Cu}_{2} \mathrm{O}$ core-shell NPs with higher $\mathrm{CuCl}_{2}$ concentration.

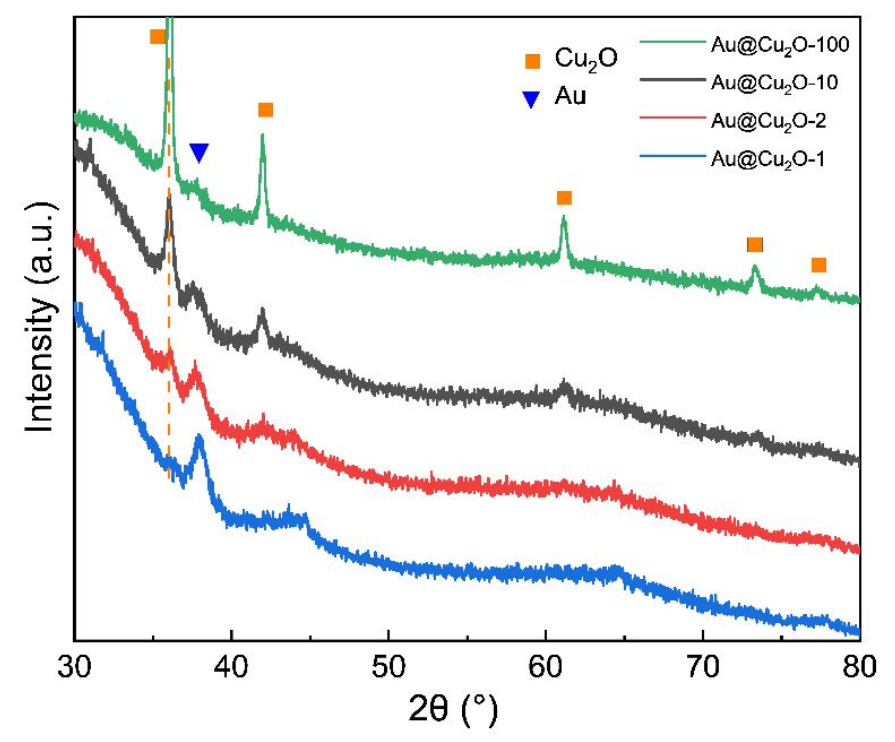

Figure S7 XRD patterns of as-synthesized $\mathrm{Au} @ \mathrm{Cu}_{2} \mathrm{O}$ core-shell nanoparticles with various shell thicknesses. 


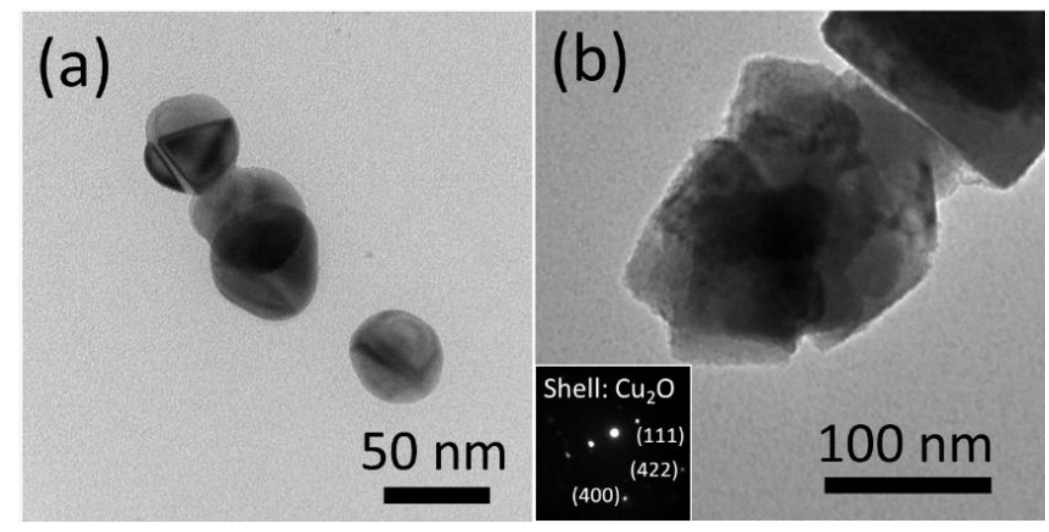

Figure S8 TEM images of as prepared (a) Ag NPs and (b) $\mathrm{Ag} @ \mathrm{Cu}_{2} \mathrm{O}$ core-shell NPs
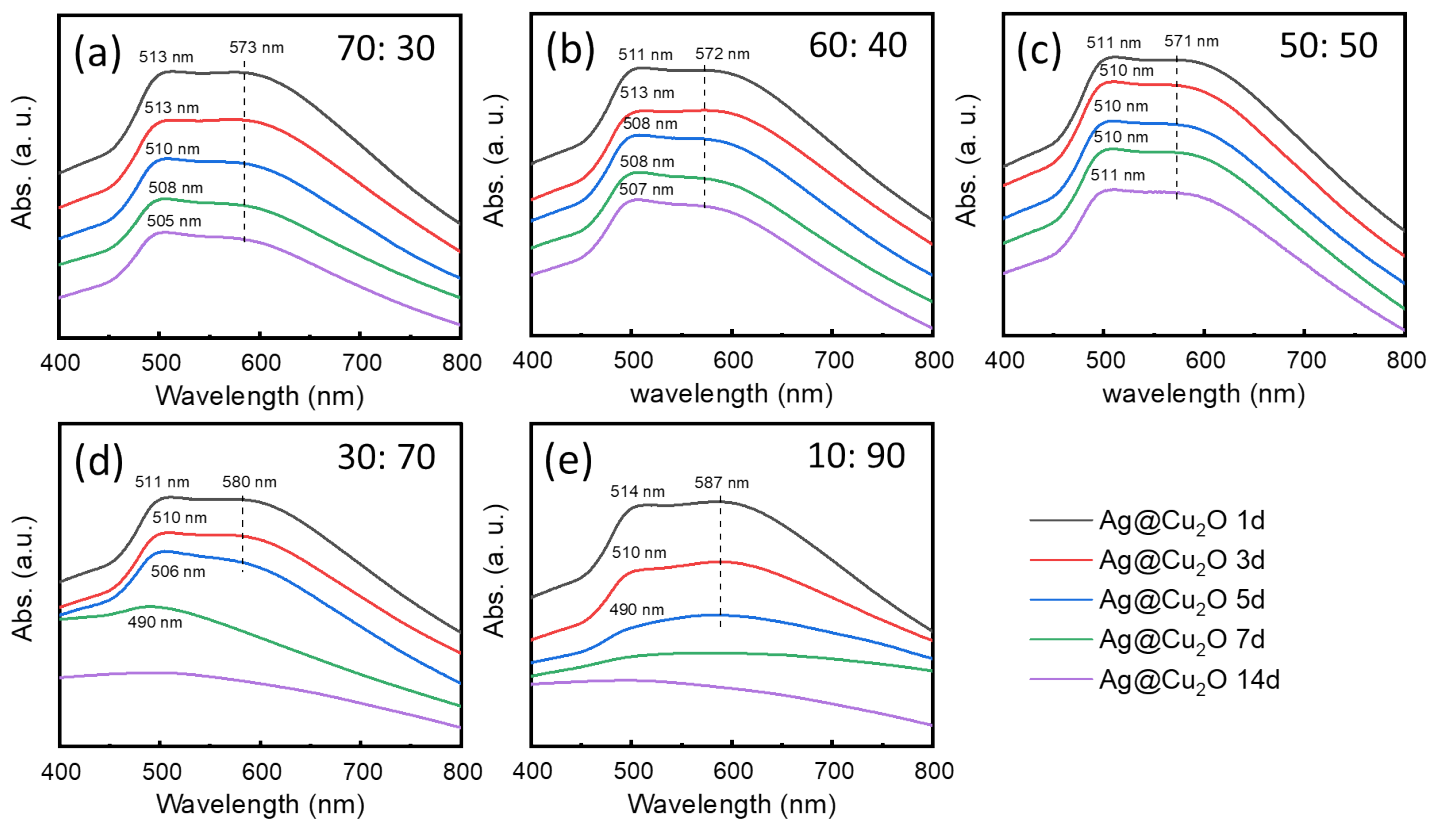

Figure S9 Degradation of $\mathrm{Ag} @ \mathrm{Cu}_{2} \mathrm{O}$ suspensions. UV-Vis absorption spectra of $\mathrm{Ag} @ \mathrm{Cu}_{2} \mathrm{O}$ core-shell NPs suspensions after synthesis for 1 day (black line), 3 days (red line), 5 days (blue line), 7 days (green line) and 14 days (purple line), stored in ethanolwater mixed solvents with compositions (ethanol:water, vol \%) of (a) 70:30, (b) 60:40, (c) 50:50, (d) 30:70 and (e) 10:90 at room temperature (set as $25^{\circ} \mathrm{C}$ ). 


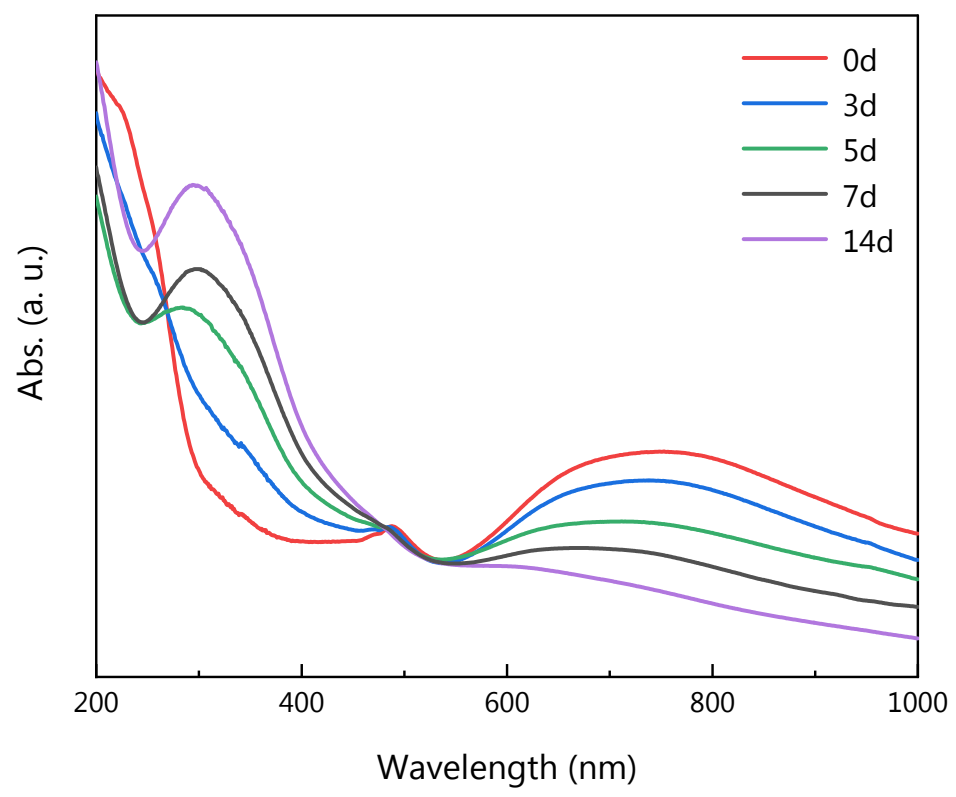

Figure S10 UV-Vis absorption spectra of $\mathrm{Cu}_{2} \mathrm{O}$ NPs stored in ethanol-water mixed solvent with composition of 30:70 in vol\% (solvent \#4).
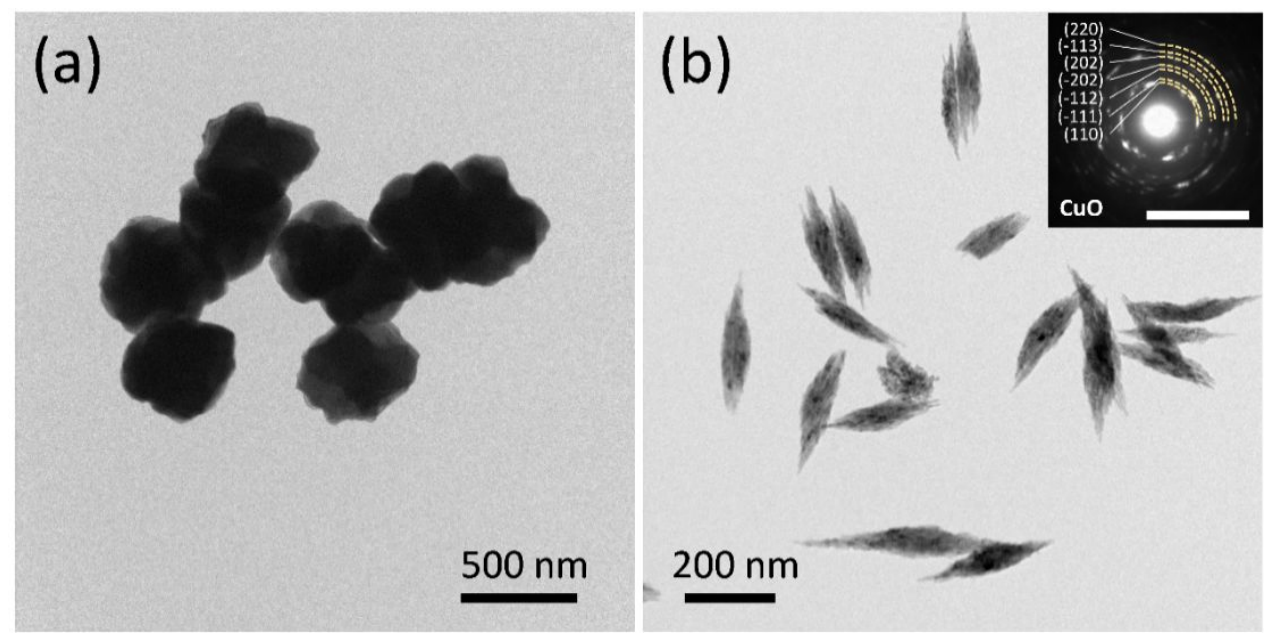

Figure $\mathrm{S11}$ TEM images of (a) as-prepared $\mathrm{Cu}_{2} \mathrm{O}$ particles and (b) $\mathrm{Cu}_{2} \mathrm{O}$ particles dispersed in 30:70 ethanol-water solvent for 30 days at room temperature. 